\title{
Strained Phase Boundaries in Li-rich Cathodes; An Atomic Resolution Study
}

\author{
Soroosh Sharifi-Asl ${ }^{1}$, Arturo Gutierrez ${ }^{2}$, Jason $\mathrm{Croy}^{2}$, Mahalingam Balasubramanian $^{3}$, Reza \\ Shahbazian-Yassar ${ }^{1 *}$ \\ 1. Mechanical and Industrial Engineering Department, University of Illinois at Chicago, Chicago, \\ Illinois, United States. \\ 2. Chemical Science and Engineering, Argonne National Laboratory, Argonne, IL, United States. \\ 3. Advanced Photon Source, Argonne National Laboratory, Argonne, IL, United States. \\ * Corresponding author: rsyassar@uic.edu
}

Li-rich NMC cathodes are considered as the next generation of Li-ion battery cathodes with capacities of $\sim 300 \mathrm{mAhg}^{-1}[1]$. However, they suffer from rapid capacity and voltage decay that is linked to their structural instability [2]. To understand the origins of such rapid degradation, the atomic structure of Li-rich cathodes should be fully comprehended. However, there is still an ongoing debate on the atomic structure of pristine Li-rich cathodes and their structure have been denoted in three opposing ways; (1) original $(\mathrm{R} \overline{3} \mathrm{~m})$ layered structure solid solution, where the extra $\mathrm{Li}$ ions are taking the transition metal sites, (2) a nano-composite of the $\mathrm{R} \overline{3} \mathrm{~m} \mathrm{LiMO}_{2}$ and the $\mathrm{C} 2 / \mathrm{m} \mathrm{Li}_{2} \mathrm{MnO}_{3}$ that is denoted as of $\mathrm{xLi}_{2} \mathrm{MnO}_{3}-(1-\mathrm{x}) \mathrm{LiMO}_{2}$, (3) a $\mathrm{C} 2 / \mathrm{m} \mathrm{Li}_{2} \mathrm{MnO}_{3}$ solid solution phase with random Li/TM mixture[3]. In our research we have utilized aberration corrected scanning transmission electron microscopy (AC-STEM) and electron energy loss spectroscopy (EELS) to elucidate the atomic configuration of Li-rich cathode materials.

The analyzed sample is made of micron-size secondary particles that are composed of primary Lirich cathode nano-particles. The individual nano-particles that are separated by ultra-sonication, are thin enough at the core areas to be electron transparent. High angle annular dark field imaging (HAADF), which is a z-contrast imaging technique and can resolve the transition metal atoms, has been utilized as the primary imaging technique in this research. By performing HAADF imaging on Li-rich cathode samples, it was observed that the primary particles are composed of different grains with various configurations (Figure 1A). Based on lattice plane measurements and fast Fourier transform (FFT) analysis (Figure 1B) the interphase can be labeled as the boundary of monoclinic $\mathrm{Li}_{2} \mathrm{MO}_{3}$ type structure with the hexagonal $\mathrm{LiMO}_{2}$ phase. Based on the energy dispersive spectroscopy (EDS) results the composition of the sample is uniform in both grains, which suggest that both monoclinic and hexagonal phases are solid solution structures. These results that were proven statistically on various particles, reveal that the nano-composite $\mathrm{xLi}_{2} \mathrm{MO}_{3}$ $(1-\mathrm{x}) \mathrm{LiMO}_{2}$ notion is the correct atomic arrangement of our Li-rich cathodes samples.

By further imaging, it was observed that the phase boundaries can have diverse configurations. For instance, Figure 1C demonstrates a monoclinic/monoclinic phase boundary that can be identified by FFT analysis. In this image, the phase boundary is parallel to the layered (010) planes of the monoclinic phases as can be clearly observed from the reconstructed color-coded inset. By performing geometric phase analysis (GPA) on this image, it can be observed that the interphase is under a significant tensile strain, as the (010) planes distance has increased from $4.82 \AA$ to $\sim 5.8$ $\AA$ at the boundary. Such large strain can result in cracking of the interphase during repeated cycling, where consecutive expansion and contraction of the lattice is expected. The identified 
strain in the phase boundaries can be considered as the underlying reason for higher susceptibility of Li-rich cathode to cracking and defect formation as detected by Bragg coherent diffractive imaging (BCDI)[4]. Moreover, another configuration of phase boundary, where the interphase is perpendicular to the layered (010)/(003) lattice of monoclinic/hexagonal grains is demonstrated in the Figure 1E. Based on the EELS analysis, such interphases have higher concentration of Ni compared to adjacent areas. because such chemical composition deviation has been identified only in the cycled sample, it has been speculated that Ni migration to the interphase occurs during the cycling and can also contribute to the degradation of the cathodes. DFT calculations are being carried out to further understand the atomistic mechanics of the identified metal diffusion process.

References:

[1]B. Qiu, M. Zhang and Y. S. Meng, Chem. Mater. 29 (2017), p. 2.

[2]M. Sathiya, A. M. Abakumov and J-M. Tarascon, Nat. Mater. 14, (2015), p 230.

[3]A. K. Shukla, Q. M. Ramasse and G. Chen, Nat. Commun. 6 (2015) p. 1.

[4] A. Singer, M. Zhang, and O. G. Shpyrko, Nat. Energy 3 (2018) P.641.

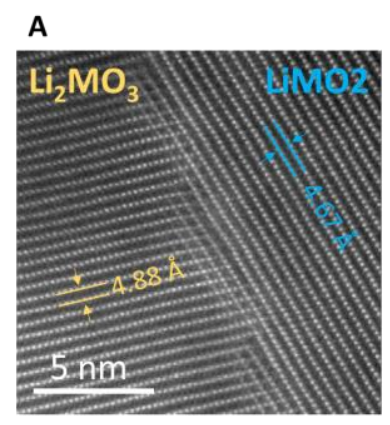

B

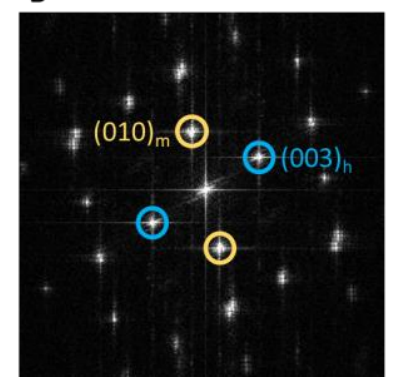

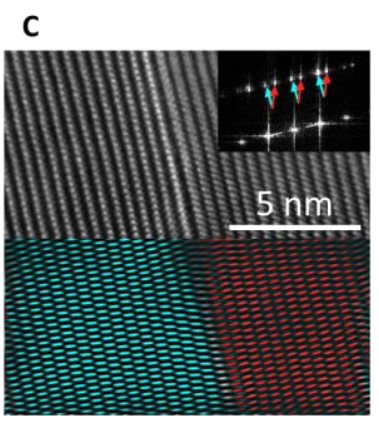

D

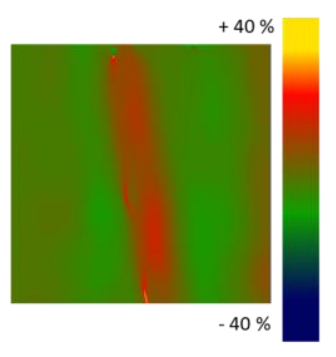

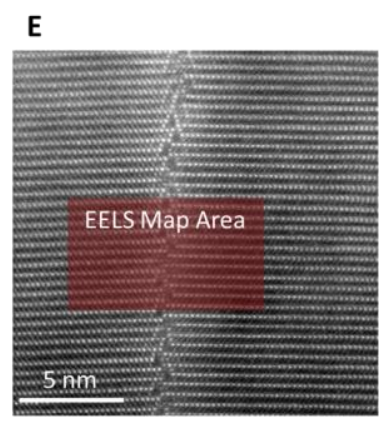

$\mathbf{F}$

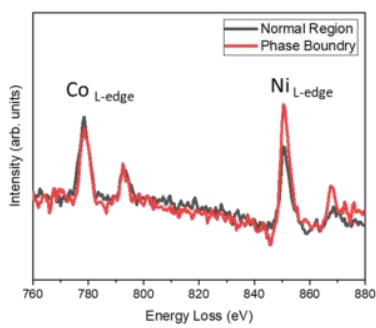

Figure 1. Comprehensive STEM/EELS analysis on the phase boundaries in Li-rich cathodes structure. (A) Atomic resolution HAADF image from a core area illustrating the boundary of monoclinic $\mathrm{Li}_{2} \mathrm{MO}_{3}$ phase and hexagonal $\mathrm{LiMO}_{2}$ phases. (B) The corresponding FFT image in which the (010) plane of the monoclinic phase and the (003) plane of the hexagonal phase is identified. (C) Atomic resolution HAADF imaging from a monoclinic/monoclinic phase boundary in a parallel configuration. Two phase structure of the image is demonstrated by the arrows on the FFT inset, based on which the color-coded inset is reconstructed. (D) Geometric phase analysis results showing the tensile strain at the phase boundary. (E) Atomic resolution HAADF image demonstrating another configuration of phase boundary where the boundary is perpendicular to the layered structure. (F) Electron energy loss spectroscopy results demonstrating the increased Ni concentration in the phase boundary. 